\title{
DETERMINATION OF THE CRITICAL LOADING AREAS ON THE DISCRETE SPHERICAL DOMES USING INFLUENCE SURFACES
}

\author{
ANita HANDRUleva \\ USEA "Lyuben Karavelov", Faculty of Construction, \\ 175, Suhodolska St., 1373 Sofia, Bulgaria, \\ e-mail: anita_handruleva@abv.bg
}

\begin{abstract}
In this article, the influence surfaces of discrete spherical domes have been investigated. Using both the discrete and Finite Element Method (FEM) for frames to generate five different models of domes, being the objects of my study, is discussed herein. Computational models differ in the type of grid configuration. Selected frames are in the meridian, parallel and diagonal directions, as for each frame an influence surface modelled by exerted vertical load to the spherical surface has been built. Research and graphical representations have been processed by using software based on the FEM, and employing static and kinematic methods. For comparison, the influence surfaces introduced the following parameters: absolute density, compressive density and tensile density. Based on the aforesaid parameters the following correlated factors have been determined: coefficient of compressive activation and coefficient of tensile activation. Results are presented in graphical and tabular form.

Keywords: spherical domes, discrete element modelling, FEM.
\end{abstract}

\section{INTRODUCTION}

Influence Surface (IS) for effort (support reaction, bending moment, shear and normal forces) is called three-dimensional graph showing the change of this magnitude as a function of the position of the moving concentrated force with a single value and constant direction, applied to the "road" surface, see [1-5].

In flat lattice systems it is a set of lines of influence, built on separate orthogonal sections with equal intervals on the surface of the investigated object. Many difficulties in the study of domes exist. The distance between sections

DOI: 10.7546/EngSci.LVII.20.03.02 
decreases in the height of the meridian, and the geometric area that simulates IS is with a double curvature and has got to find a "readable" form for their presentation. The question is: "How to be an IS displayed? Either as a projection on a horizontal plane that illustrates insufficiently readable the change of IS in the areas around the supporting ring (base supports), or via a cartographic projection by unfolding of the spherical surface onto a vertical plane?" In this case the distances in-between the meridians at/around the top of the dome appear to be greatly exaggerated, thence the surface gets deformed. A third option is a perspective presentation of the dome in a selected view, as the ordinates of the influence surface are applied linear-vertically. This option is adopted for further submission. The manual calculation of the ordinates of this graph for space systems by means of method of displacements and method of forces is almost impossible due to the high static indeterminateness, see [6-10]. The calculation of the ordinates of an IS for a particular exerted effort by using advanced software programs on Finite Element Method (FEM) (SAP2000, ANSYS, etc.) has been really improved, which in turn facilitates the entire object- or element-based modelling process, see [11-13]. As well-known as it is from Structural Mechanics, there are three methods for the construction of lines of influence that can be applied to build the IS: static, kinematic and a combined one.

The altering of the studied parameters in value and sign has been approached by applying the static method when changing the location of the single moving force in the defined road sections. For each position of the simulated force exerted, an equation of equilibrium is recorded, and (searched) an assumed response effort is calculated, etc.

The kinematic method is based on the principle of possible displacement, whereat the equations drawn for the work of all forces acting on the system, previously converted into a mechanism by removing the link, or supporting the effort, are recorded.

Influence Surfaces have been used to study the domes in the stage of limit equilibrium (loss of stability) and in the study of loss of bearing capacity of the elements.

\section{MAIN OBJECTIVES AND TASKS RELATED TO THE STUDY OF THE INFLUENCE SURFACES}

- To outline areas for obtaining extreme (compressive and tensile) values for efforts in the bars of the dome with different morphology of the grid configuration under the action of vertical load to the spherical surface;

Engineering Sciences, LVII, 2020, No. 3 
- To identify areas of IS in which the load does not affect significantly on a rod test effort, which would simplify the procedure for seeking extreme efforts;

- To clarify the role of symmetric, asymmetric and local load on the state of the rods.

\section{DESCRIPTION OF SELECTED COMPUTATIONAL MODELS}

As objects to study, the surfaces of influence in lattice structures are considered as representatives of the five domes. The domes are conditionally named K.1 $\div$ K.5, respectively, as shown in Fig. 1 .
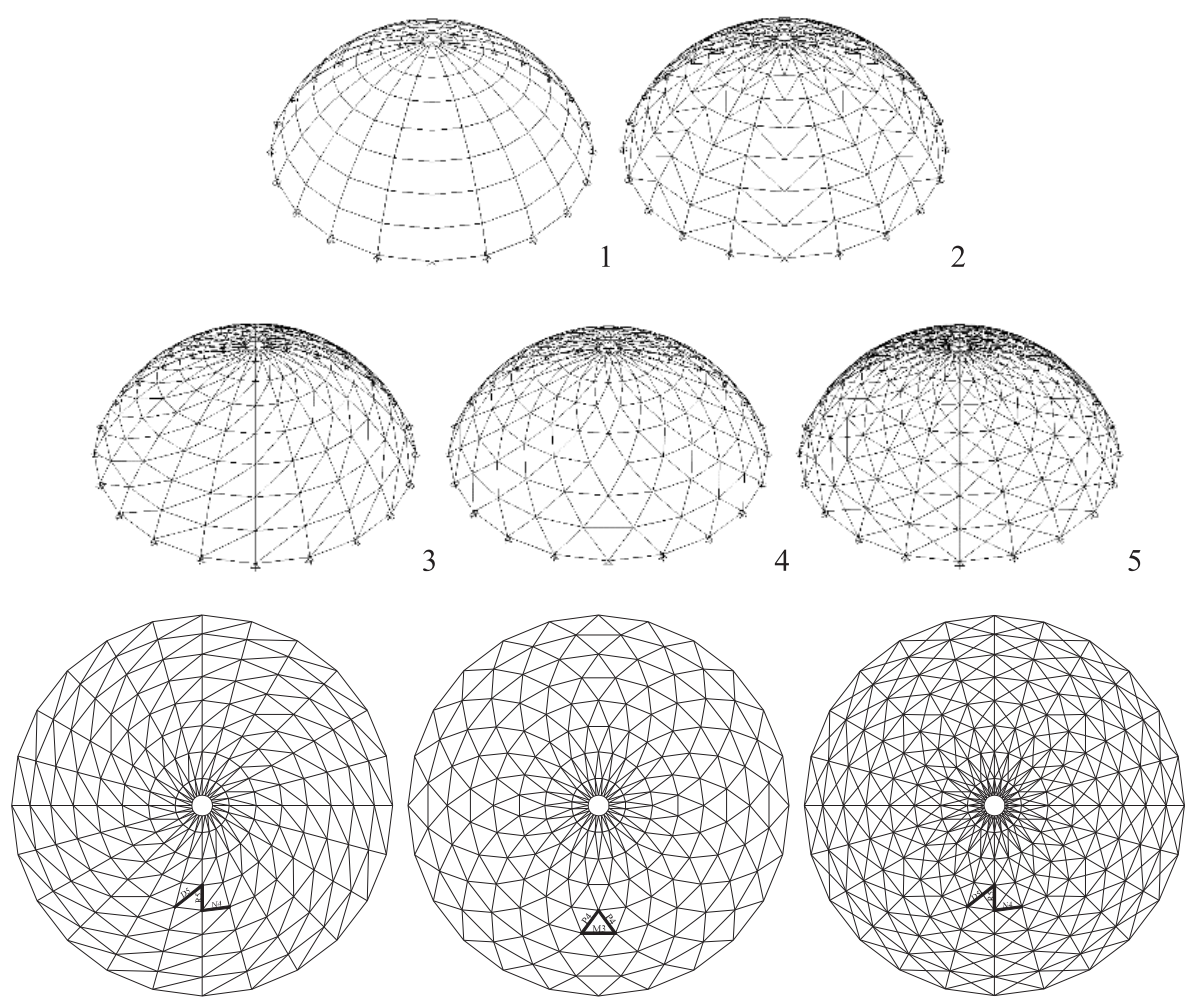

Fig. 1. Selected representatives of the single layer spherical domes

To generate the models the following fixed parameters have been adapted, see Fig. 2: 


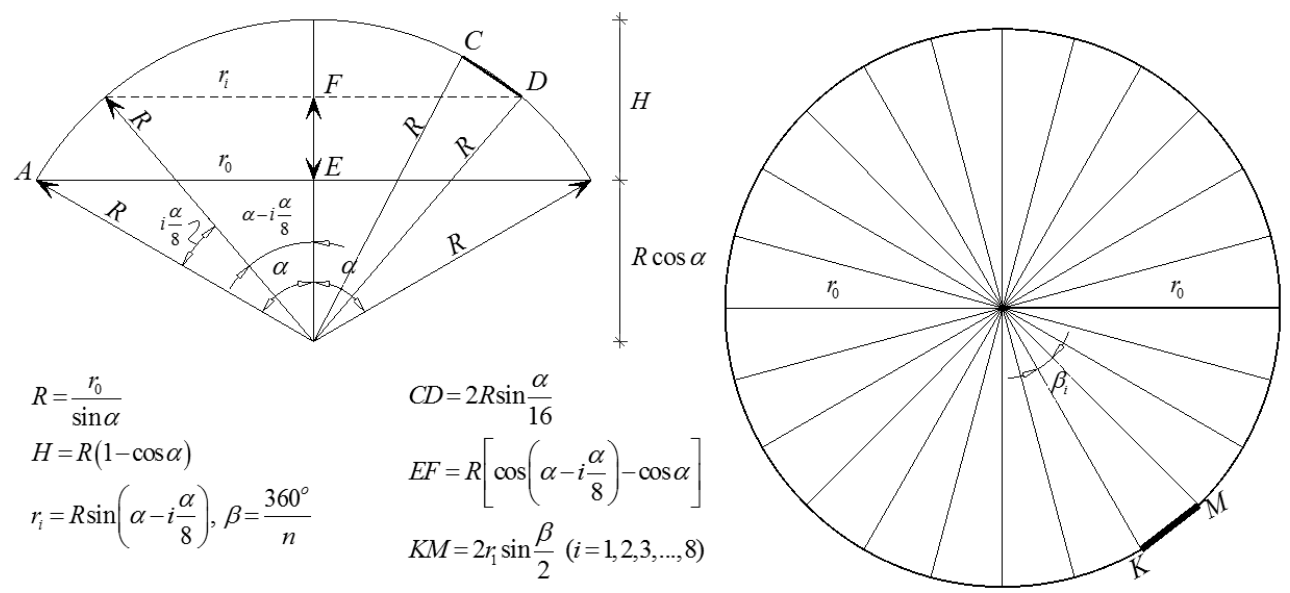

Fig. 2. Geometrical characteristics of numerical models

- the diameter of the base $D=30 \mathrm{~m}$;

- a diameter of the ring at the top $r_{k}=1.65 \mathrm{~m}$;

- central semi angle $50^{\circ}$ with the corresponding height $7 \mathrm{~m}$;

- a cyclic angle at the base $\theta=15^{\circ}$;

- number of parallels separating meridian lines (inner rings) $n=7$;

- rigid connection between the elements of the grid structure;

- the domes have radially movable supports;

- one-dimensional finite elements of a Frame type were employed to generate the computational models of single-layer discrete spherical domes;

- For the structural elements, cross-sections of pipe profiles have been chosen, namely: $\varnothing 83 \times 4$ of $A=9.93 \mathrm{~cm}^{2}$ area, a moment of inertia $I=77.64 \mathrm{~cm}^{4}$ and a radius of inertia $i=2.8 \mathrm{~cm}$. The profiles are made of steel class S235JRH following EN 10219-2 with a calculated resistance of steel 235 MPa.

For all elements of the studied domes, tubular profiles with equal geometric characteristics of the cross-section have been adapted.

Bars in the meridian and parallel direction, and diagonals are selected. For every one of them, an IS of vertical load to the spherical surface is built. In meridian direction, elements are conventionally denoted by $\mathrm{R} 1 \div \mathrm{R} 8$, in the ring (parallel) - with $\mathrm{N} 1 \div \mathrm{N} 7$, and diagonals - with $\mathrm{D} 1 \div \mathrm{D} 8$.

The study and graphic imaging have been done with a SAP_2000 (version 15) software based on the FEM using both the static and kinematic method. 


\section{COMPARATIVE ANALYSIS OF THE RESULTS}

Figures 3 through 7 show the spatial images of the influence surface of the axial force for a selected element.
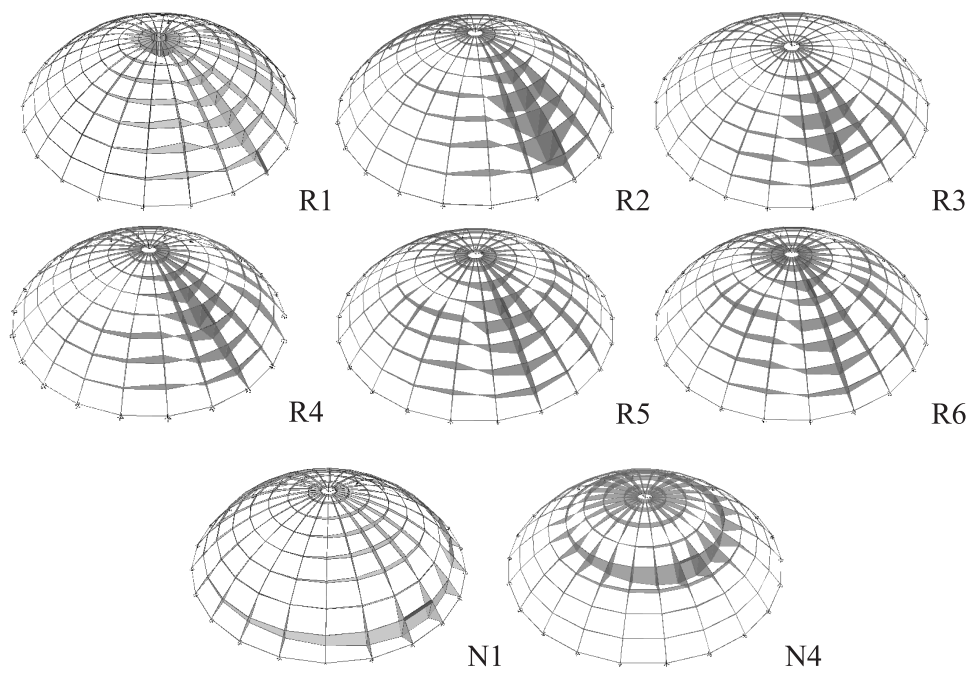

Fig. 3. Influence surface for normal effort built upon a static method for dome K.1
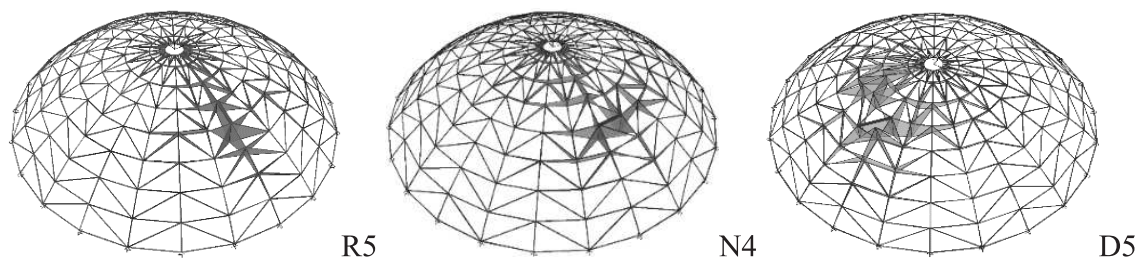

Fig. 4. Influence surface for normal effort built upon a static method for dome K.2
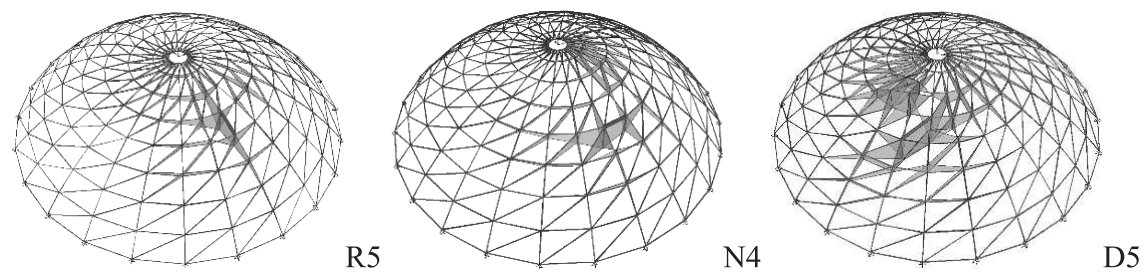

Fig. 5. Influence surface for normal effort, built upon a static method for dome K.3 

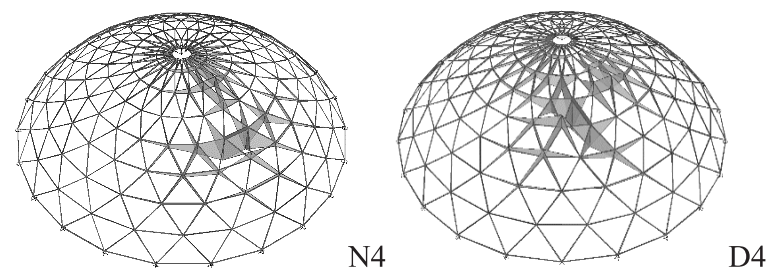

Fig. 6. Influence surface for normal effort built upon a static method for dome K.4
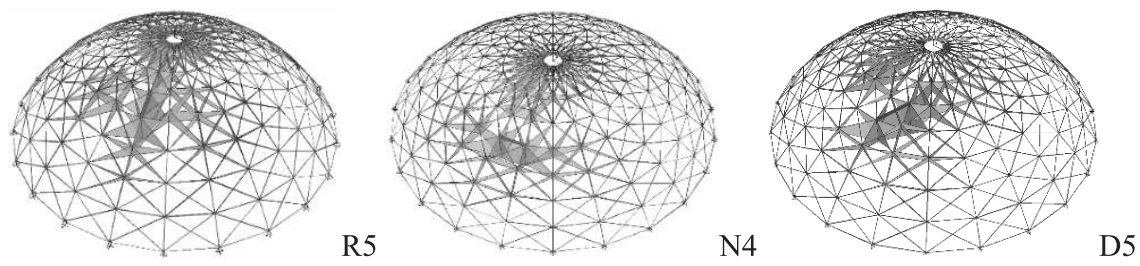

Fig. 7. Influence surface for normal effort built upon a static method for dome K.5

In the Figures from 8 to 11 another approach has been used to illustrate the influence surface for a normal force by the application of the kinematic method. In this visualization, the areas of tensile and compressive activation in the studied element are clearly outlined/articulated.

The kinematic method is based on the principle of possible displacements. In this method, equations for the work of the forces acting on the system have been recorded. It was turned into a mechanism by removing the connection $R$, supporting the effort. As well known, following the principle of possible displacements $F \cdot \bar{\delta}_{f}+R . \bar{\delta}_{R}=0$.

When $F=1$ we obtain:

$$
R=-\frac{\bar{\delta}_{f}}{\bar{\delta}_{R}}
$$

where $\bar{\delta}_{f}$ is the displacement of the application point of moving force, decomposed in its direction, i.e. combination of decomposed displacements of all crawled by force $F$ points on the way; $\bar{\delta}_{R}$ is the mutual displacement in the points of application of force $R$, decomposed in its direction.

At possible displacement $\bar{\delta}_{R}=-1$ the surface of decomposed displacements would be identical to the surface of the influence of the effort: " $R$ " $=" \bar{\delta}_{f}$ ".

In other words, the influence surface is defined as elastic surface - diagram of the displacements of the points of the nodes of the structure in the negative single mutual displacement or rotation in the direction of the effort. 

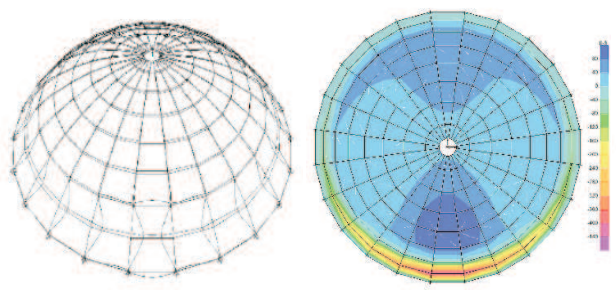

(a)

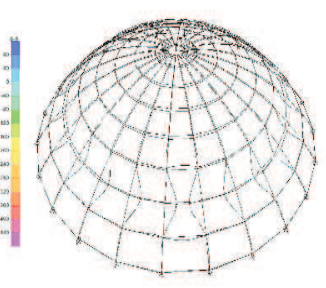

(b)

Fig. 8. Influence surface for normal effort in element from dome K.1 built employing the kinematic method: (a) N1; (b) N2
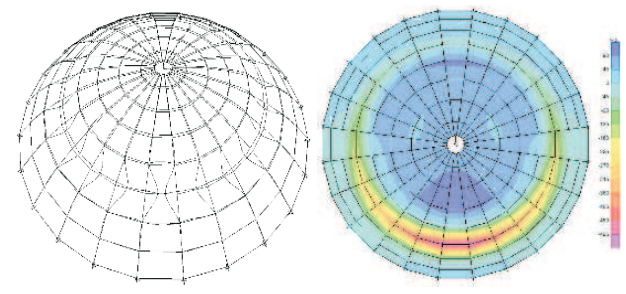

(a)
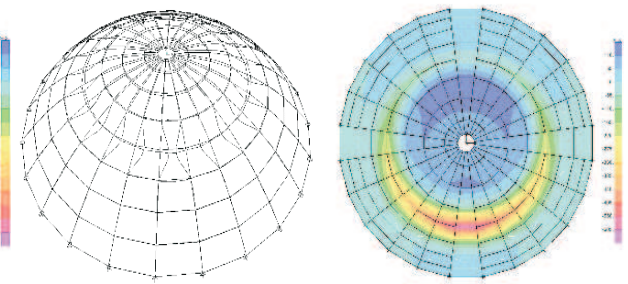

(b)

Fig. 9. Influence surface for normal effort in element from dome K.1 built using the kinematic method: (a) N3; (b) N4
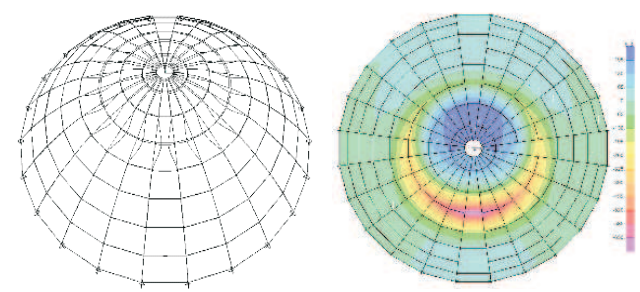

(a)
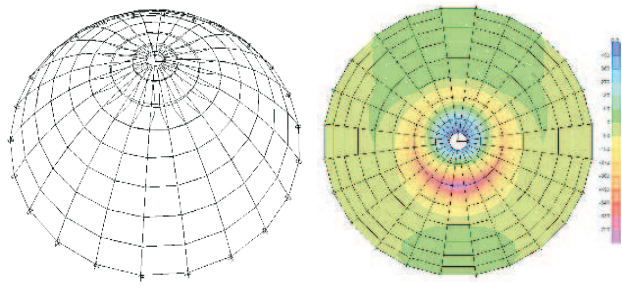

(b)

Fig. 10. Influence surface for normal effort in element from dome K.1 built using the kinematic method: (a) N5; (b) N6
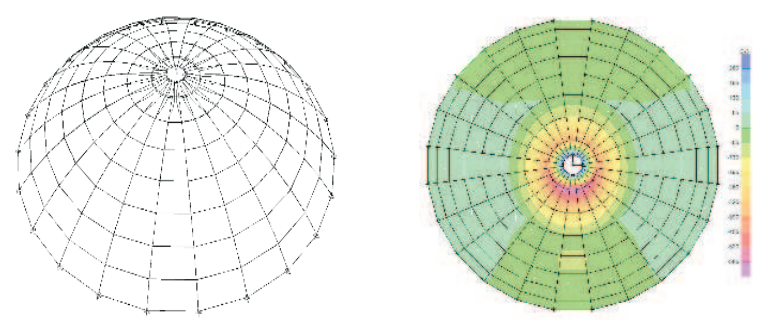

Fig. 11. Influence surface for normal effort in element N7 from dome K.1, built using the kinematic method 
After processing the results (in the case of ordinates $\eta_{i}$ of the influence surface) for the evaluation of IS for efforts in the elements from different computational models following new evaluative-quantitative indicators have been introduced. See Tables $1 \div 13$ :

- Absolute density $D_{a}=\sum\left|\eta_{i}\right|$;

- Compression density $D_{c}=\sum\left(-\eta_{i}\right)$;

Table 1. Evaluative-quantitative indicators and correlated coefficients $\left[{ }^{*} \ldots\right]$ of elements $\mathrm{N} 1 \div \mathrm{N} 8$ from dome K.1

\begin{tabular}{|c|c|c|c|c|c|c|c|c|}
\hline $\begin{array}{c}\text { Dome } \\
\text { K.1 }\end{array}$ & $\begin{array}{c}\text { Element } \\
\text { N1 }\end{array}$ & $\begin{array}{c}\text { Element } \\
\text { N2 }\end{array}$ & $\begin{array}{c}\text { Element } \\
\text { N3 }\end{array}$ & $\begin{array}{c}\text { Element } \\
\text { N4 }\end{array}$ & $\begin{array}{c}\text { Element } \\
\text { N5 }\end{array}$ & $\begin{array}{c}\text { Element } \\
\text { N6 }\end{array}$ & $\begin{array}{c}\text { Element } \\
\text { N7 }\end{array}$ & $\begin{array}{c}\text { Element } \\
\text { N8 }\end{array}$ \\
\hline$D_{a}$ & 10.0409 & 10.7765 & 13.6341 & 17.1060 & 22.3683 & 28.2988 & 23.4068 & 55.2918 \\
\hline$D_{c}$ & 3.3686 & 4.4642 & 5.6479 & 7.4039 & 9.5027 & 11.8863 & 15.3172 & 47.2442 \\
\hline$D_{t}$ & 6.6723 & 6.3123 & 7.9862 & 9.7021 & 12.8656 & 16.4126 & 8.0896 & 8.0476 \\
\hline$K_{c}$ & 0.3355 & 0.4143 & 0.4142 & 0.4328 & 0.4248 & 0.4200 & 0.6544 & 0.8545 \\
\hline$K_{t}$ & 0.6645 & 0.5857 & 0.5858 & 0.5672 & 0.5752 & 0.5800 & 0.3456 & 0.1455 \\
\hline
\end{tabular}

Table 2. Evaluative-quantitative indicators and correlated coefficients $[* \ldots]$ of elements $\mathrm{R} 1 \div \mathrm{R} 8$ from dome K.1

\begin{tabular}{|c|c|c|c|c|c|c|c|c|}
\hline $\begin{array}{c}\text { Dome } \\
\text { K.1 }\end{array}$ & $\begin{array}{c}\text { Element } \\
\text { R1 }\end{array}$ & $\begin{array}{c}\text { Element } \\
\text { R2 }\end{array}$ & $\begin{array}{c}\text { Element } \\
\text { R3 }\end{array}$ & $\begin{array}{c}\text { Element } \\
\text { R4 }\end{array}$ & $\begin{array}{c}\text { Element } \\
\text { R5 }\end{array}$ & $\begin{array}{c}\text { Element } \\
\text { R6 }\end{array}$ & $\begin{array}{c}\text { Element } \\
\text { R7 }\end{array}$ & $\begin{array}{c}\text { Element } \\
\text { R8 }\end{array}$ \\
\hline$D_{a}$ & 17.2045 & 17.2399 & 17.4409 & 18.4791 & 20.1780 & 22.3439 & 23.9732 & 19.3688 \\
\hline$D_{c}$ & 14.0631 & 14.0416 & 14.0337 & 14.5614 & 15.4723 & 16.8190 & 18.1104 & 14.8186 \\
\hline$D_{t}$ & 3.1414 & 3.1983 & 3.4072 & 3.9177 & 4.7057 & 5.5249 & 5.8628 & 4.5502 \\
\hline$K_{c}$ & 0.8174 & 0.8145 & 0.8046 & 0.7880 & 0.7668 & 0.7527 & 0.7554 & 0.7651 \\
\hline$K_{t}$ & 0.1826 & 0.1855 & 0.1954 & 0.2120 & 0.2332 & 0.2473 & 0.2446 & 0.2349 \\
\hline
\end{tabular}

Table 3. Evaluative-quantitative indicators and correlated coefficients $\left[{ }^{*} \ldots\right]$ of elements $\mathrm{N} 1 \div \mathrm{N} 8$ from dome K.2

\begin{tabular}{|c|c|c|c|c|c|c|c|c|}
\hline $\begin{array}{c}\text { Dome } \\
\text { K.2 }\end{array}$ & $\begin{array}{c}\text { Element } \\
\text { N1 }\end{array}$ & $\begin{array}{c}\text { Element } \\
\text { N2 }\end{array}$ & $\begin{array}{c}\text { Element } \\
\text { N3 }\end{array}$ & $\begin{array}{c}\text { Element } \\
\text { N4 }\end{array}$ & $\begin{array}{c}\text { Element } \\
\text { N5 }\end{array}$ & $\begin{array}{c}\text { Element } \\
\text { N6 }\end{array}$ & $\begin{array}{c}\text { Element } \\
\text { N7 }\end{array}$ & $\begin{array}{c}\text { Element } \\
\text { N8 }\end{array}$ \\
\hline$D_{a}$ & 13.9494 & 15.2844 & 17.5825 & 20.3577 & 25.1654 & 32.6626 & 28.0829 & 50.7764 \\
\hline$D_{c}$ & 5.4331 & 6.5678 & 7.5960 & 8.9871 & 10.8107 & 14.1039 & 17.5656 & 44.3627 \\
\hline$D_{t}$ & 8.5164 & 8.7166 & 9.9865 & 11.3706 & 14.3547 & 18.6237 & 10.5173 & 6.4137 \\
\hline$K_{c}$ & 0.3895 & 0.4297 & 0.4320 & 0.4415 & 0.4296 & 0.4318 & 0.6255 & 0.8737 \\
\hline$K_{t}$ & 0.6105 & 0.5703 & 0.5680 & 0.5585 & 0.5704 & 0.5702 & 0.3745 & 0.1263 \\
\hline
\end{tabular}


Table 4. Evaluative-quantitative indicators and correlated coefficients [*...] of elements $\mathrm{R} 1 \div \mathrm{R} 8$ from dome $\mathrm{K} .2$

\begin{tabular}{|c|c|c|c|c|c|c|c|c|}
\hline $\begin{array}{c}\text { Dome } \\
\text { K.2 }\end{array}$ & $\begin{array}{c}\text { Element } \\
\text { R1 }\end{array}$ & $\begin{array}{c}\text { Element } \\
\text { R2 }\end{array}$ & $\begin{array}{c}\text { Element } \\
\text { R3 }\end{array}$ & $\begin{array}{c}\text { Element } \\
\text { R4 }\end{array}$ & $\begin{array}{c}\text { Element } \\
\text { R5 }\end{array}$ & $\begin{array}{c}\text { Element } \\
\text { R6 }\end{array}$ & $\begin{array}{c}\text { Element } \\
\text { R7 }\end{array}$ & $\begin{array}{c}\text { Element } \\
\text { R8 }\end{array}$ \\
\hline$D_{a}$ & 10.9198 & 12.1115 & 14.6621 & 16.2071 & 16.9858 & 17.4847 & 19.1164 & 16.3471 \\
\hline$D_{c}$ & 10.8441 & 10.8724 & 12.3520 & 13.1906 & 13.7025 & 14.2277 & 15.4805 & 14.0620 \\
\hline$D_{t}$ & 0.0757 & 1.2391 & 2.3100 & 3.0164 & 3.2833 & 3.2570 & 3.6358 & 2.2851 \\
\hline$K_{c}$ & 0.9931 & 0.8977 & 0.8424 & 0.8139 & 0.8067 & 0.8137 & 0.8098 & 0.8602 \\
\hline$K_{t}$ & 0.0069 & 0.1023 & 0.1576 & 0.1861 & 0.1933 & 0.1863 & 0.1902 & 0.1398 \\
\hline
\end{tabular}

Table 5. Evaluative-quantitative indicators and correlated coefficients [*...] of elements D $\div$ D 8 from dome K.2

\begin{tabular}{|c|c|c|c|c|c|c|c|c|}
\hline $\begin{array}{c}\text { Dome } \\
\text { K.2 }\end{array}$ & $\begin{array}{c}\text { Element } \\
\text { D1 }\end{array}$ & $\begin{array}{c}\text { Element } \\
\text { D2 }\end{array}$ & $\begin{array}{c}\text { Element } \\
\text { D3 }\end{array}$ & $\begin{array}{c}\text { Element } \\
\text { D4 }\end{array}$ & $\begin{array}{c}\text { Element } \\
\text { D5 }\end{array}$ & $\begin{array}{c}\text { Element } \\
\text { D6 }\end{array}$ & $\begin{array}{c}\text { Element } \\
\text { D7 }\end{array}$ & $\begin{array}{c}\text { Element } \\
\text { D8 }\end{array}$ \\
\hline$D_{a}$ & 12.1226 & 13.0328 & 14.6688 & 16.7523 & 19.1577 & 22.3267 & 24.4963 & 26.0024 \\
\hline$D_{c}$ & 5.4328 & 6.8346 & 7.3951 & 5.4357 & 9.6013 & 11.2302 & 13.1852 & 13.9198 \\
\hline$D_{t}$ & 6.6997 & 6.1981 & 7.2736 & 8.3166 & 9.5565 & 11.0965 & 11.3111 & 12.0826 \\
\hline$K_{c}$ & 0.4482 & 0.5244 & 0.5041 & 0.3245 & 0.5012 & 0.5030 & 0.5383 & 0.5353 \\
\hline$K_{t}$ & 0.5527 & 0.4756 & 0.4959 & 0.4964 & 0.4988 & 0.4970 & 0.4617 & 0.4647 \\
\hline
\end{tabular}

Table 6. Evaluative-quantitative indicators and correlated coefficients $\left[{ }^{*} \ldots\right]$ of elements $\mathrm{N} 1 \div \mathrm{N} 8$ from dome $\mathrm{K} .3$

\begin{tabular}{|c|c|c|c|c|c|c|c|c|}
\hline $\begin{array}{c}\text { Dome } \\
\text { K.3 }\end{array}$ & $\begin{array}{c}\text { Element } \\
\text { N1 }\end{array}$ & $\begin{array}{c}\text { Element } \\
\text { N2 }\end{array}$ & $\begin{array}{c}\text { Element } \\
\text { N3 }\end{array}$ & $\begin{array}{c}\text { Element } \\
\text { N4 }\end{array}$ & $\begin{array}{c}\text { Element } \\
\text { N5 }\end{array}$ & $\begin{array}{c}\text { Element } \\
\text { N6 }\end{array}$ & $\begin{array}{c}\text { Element } \\
\text { N7 }\end{array}$ & $\begin{array}{c}\text { Element } \\
\text { N8 }\end{array}$ \\
\hline$D_{a}$ & 13.7855 & 15.1941 & 17.5804 & 20.3818 & 25.0989 & 33.4057 & 24.6515 & 49.8503 \\
\hline$D_{c}$ & 5.1478 & 6.7076 & 7.6527 & 9.0062 & 10.7678 & 14.6477 & 16.0271 & 43.9952 \\
\hline$D_{t}$ & 8.6377 & 8.4865 & 9.9278 & 11.3756 & 14.3311 & 18.7580 & 8.6243 & 5.8551 \\
\hline$K_{c}$ & 0.3734 & 0.4415 & 0.4353 & 0.4419 & 0.4290 & 0.4385 & 0.6501 & 0.8825 \\
\hline$K_{t}$ & 0.6266 & 0.5585 & 0.5647 & 0.5581 & 0.5710 & 0.5615 & 0.3499 & 0.1175 \\
\hline
\end{tabular}

Table 7. Evaluative-quantitative indicators and correlated coefficients $\left[{ }^{*} \ldots\right]$ of elements $\mathrm{R} 1 \div \mathrm{R} 8$ from dome $\mathrm{K} .3$

\begin{tabular}{|c|c|c|c|c|c|c|c|c|}
\hline $\begin{array}{c}\text { Dome } \\
\text { K.3 }\end{array}$ & $\begin{array}{c}\text { Element } \\
\text { R1 }\end{array}$ & $\begin{array}{c}\text { Element } \\
\text { R2 }\end{array}$ & $\begin{array}{c}\text { Element } \\
\text { R3 }\end{array}$ & $\begin{array}{c}\text { Element } \\
\text { R4 }\end{array}$ & $\begin{array}{c}\text { Element } \\
\text { R5 }\end{array}$ & $\begin{array}{c}\text { Element } \\
\text { R6 }\end{array}$ & $\begin{array}{c}\text { Element } \\
\text { R7 }\end{array}$ & $\begin{array}{c}\text { Element } \\
\text { R8 }\end{array}$ \\
\hline$D_{a}$ & 13.5967 & 15.7131 & 17.5510 & 19.4723 & 21.7993 & 24.2707 & 23.2571 & 22.0172 \\
\hline$D_{c}$ & 12.2456 & 13.2787 & 14.0800 & 15.0363 & 16.2600 & 17.7614 & 17.5627 & 15.4309 \\
\hline$D_{t}$ & 1.3512 & 2.4344 & 3.4710 & 4.4360 & 5.5393 & 6.5093 & 5.6944 & 6.5863 \\
\hline$K_{c}$ & 0.9006 & 0.8451 & 0.8022 & 0.7722 & 0.7459 & 0.7318 & 0.7552 & 0.7009 \\
\hline$K_{t}$ & 0.0994 & 0.1549 & 0.1978 & 0.2278 & 0.2541 & 0.2682 & 0.2448 & 0.2991 \\
\hline
\end{tabular}


Table 8. Evaluative-quantitative indicators and correlated coefficients [*...] of elements D $1 \div$ D8 from dome K.3

\begin{tabular}{|c|c|c|c|c|c|c|c|c|}
\hline $\begin{array}{c}\text { Dome } \\
\text { K.3 }\end{array}$ & $\begin{array}{c}\text { Element } \\
\text { D1 }\end{array}$ & $\begin{array}{c}\text { Element } \\
\text { D2 }\end{array}$ & $\begin{array}{c}\text { Element } \\
\text { D3 }\end{array}$ & $\begin{array}{c}\text { Element } \\
\text { D4 }\end{array}$ & $\begin{array}{c}\text { Element } \\
\text { D5 }\end{array}$ & $\begin{array}{c}\text { Element } \\
\text { D6 }\end{array}$ & $\begin{array}{c}\text { Element } \\
\text { D7 }\end{array}$ & $\begin{array}{c}\text { Element } \\
\text { D8 }\end{array}$ \\
\hline$D_{a}$ & 11.7705 & 12.9569 & 14.6666 & 16.6465 & 19.0829 & 21.9382 & 21.3920 & 20.6520 \\
\hline$D_{c}$ & 5.8715 & 6.4817 & 7.3353 & 8.3249 & 9.5509 & 10.9880 & 10.8271 & 10.9372 \\
\hline$D_{t}$ & 5.8990 & 6.4752 & 7.3313 & 8.3216 & 9.5320 & 10.9502 & 10.5649 & 9.7148 \\
\hline$K_{c}$ & 0.4988 & 0.5003 & 0.5001 & 0.5001 & 0.5005 & 0.5009 & 0.5061 & 0.5296 \\
\hline$K_{t}$ & 0.5012 & 0.4997 & 0.4999 & 0.4999 & 0.4995 & 0.4991 & 0.4939 & 0.4704 \\
\hline
\end{tabular}

Table 9. Evaluative-quantitative indicators and correlated coefficients $\left[{ }^{*} \ldots\right]$ of elements $\mathrm{M} 1 \div \mathrm{M} 8$ from dome $\mathrm{K} .4$

\begin{tabular}{|c|c|c|c|c|c|c|c|c|}
\hline $\begin{array}{c}\text { Dome } \\
\text { K.4 }\end{array}$ & $\begin{array}{c}\text { Element } \\
\text { M1 }\end{array}$ & $\begin{array}{c}\text { Element } \\
\text { M2 }\end{array}$ & $\begin{array}{c}\text { Element } \\
\text { M3 }\end{array}$ & $\begin{array}{c}\text { Element } \\
\text { M4 }\end{array}$ & $\begin{array}{c}\text { Element } \\
\text { M5 }\end{array}$ & $\begin{array}{c}\text { Element } \\
\text { M6 }\end{array}$ & $\begin{array}{c}\text { Element } \\
\text { M7 }\end{array}$ & $\begin{array}{c}\text { Element } \\
\text { M8 }\end{array}$ \\
\hline$D_{a}$ & 15.0321 & 15.9238 & 17.7497 & 20.1321 & 25.0068 & 33.7270 & 24.8276 & 49.5665 \\
\hline$D_{c}$ & 3.6188 & 5.0722 & 6.1157 & 7.5480 & 9.6862 & 13.9915 & 15.5381 & 43.8742 \\
\hline$D_{t}$ & 11.4133 & 10.8517 & 11.6340 & 12.5841 & 15.3205 & 19.7355 & 9.2895 & 5.6923 \\
\hline$K_{c}$ & 0.2407 & 0.3185 & 0.3446 & 0.3749 & 0.3873 & 0.4148 & 0.6258 & 0.8852 \\
\hline$K_{t}$ & 0.7593 & 0.6815 & 0.6554 & 0.6251 & 0.6127 & 0.5852 & 0.3742 & 0.1148 \\
\hline
\end{tabular}

Table 10. Evaluative-quantitative indicators and correlated coefficients $\left[{ }^{*} \ldots\right]$ of elements $\mathrm{P} 1 \div \mathrm{P} 8$ from dome $\mathrm{K} .4$

\begin{tabular}{|c|c|c|c|c|c|c|c|c|}
\hline $\begin{array}{c}\text { Dome } \\
\text { K.4 }\end{array}$ & $\begin{array}{c}\text { Element } \\
\text { P1 }\end{array}$ & $\begin{array}{c}\text { Element } \\
\text { P2 }\end{array}$ & $\begin{array}{c}\text { Element } \\
\text { P3 }\end{array}$ & $\begin{array}{c}\text { Element } \\
\text { P4 }\end{array}$ & $\begin{array}{c}\text { Element } \\
\text { P5 }\end{array}$ & $\begin{array}{c}\text { Element } \\
\text { P6 }\end{array}$ & $\begin{array}{c}\text { Element } \\
\text { P7 }\end{array}$ & $\begin{array}{c}\text { Element } \\
\text { P8 }\end{array}$ \\
\hline$D_{a}$ & 12.4551 & 14.1464 & 15.4310 & 16.8028 & 18.6490 & 21.3389 & 21.4709 & 20.9030 \\
\hline$D_{c}$ & 9.8312 & 10.5013 & 10.9141 & 11.4367 & 12.2578 & 13.6313 & 13.8274 & 13.0120 \\
\hline$D_{t}$ & 2.6239 & 3.6451 & 4.5169 & 5.3661 & 6.3913 & 7.7076 & 7.6435 & 7.8910 \\
\hline$K_{c}$ & 0.7893 & 0.7423 & 0.7073 & 0.6806 & 0.6573 & 0.6388 & 0.6440 & 0.6225 \\
\hline$K_{t}$ & 0.2107 & 0.2577 & 0.2927 & 0.3194 & 0.3427 & 0.3612 & 0.3560 & 0.3775 \\
\hline
\end{tabular}

Table 11. Evaluative-quantitative indicators and correlated coefficients $\left[{ }^{*} \ldots\right]$ of elements $\mathrm{N} 1 \div \mathrm{N} 8$ from dome K.5

\begin{tabular}{|c|c|c|c|c|c|c|c|c|}
\hline $\begin{array}{c}\text { Dome } \\
\text { K.5 }\end{array}$ & $\begin{array}{c}\text { Element } \\
\mathrm{N} 1\end{array}$ & $\begin{array}{c}\text { Element } \\
\mathrm{N} 2\end{array}$ & $\begin{array}{c}\text { Element } \\
\mathrm{N} 3\end{array}$ & $\begin{array}{c}\text { Element } \\
\text { N4 }\end{array}$ & $\begin{array}{c}\text { Element } \\
\text { N5 }\end{array}$ & $\begin{array}{c}\text { Element } \\
\text { N6 }\end{array}$ & $\begin{array}{c}\text { Element } \\
\text { N7 }\end{array}$ & $\begin{array}{c}\text { Element } \\
\text { N8 }\end{array}$ \\
\hline$D_{a}$ & 5.946866 & 9.7083 & 12.5719 & 16.1382 & 22.3229 & 31.0855 & 26.4422 & 50.9588 \\
\hline$D_{c}$ & 2.7507 & 3.2219 & 4.1863 & 5.7485 & 7.9730 & 11.6697 & 15.4092 & 44.5902 \\
\hline$D_{t}$ & 3.1962 & 6.4864 & 8.3856 & 10.3898 & 14.3499 & 19.4158 & 11.0330 & 6.3686 \\
\hline$K_{c}$ & 0.4625 & 0.3319 & 0.3330 & 0.3562 & 0.3572 & 0.3754 & 0.5827 & 0.8750 \\
\hline$K_{t}$ & 0.5375 & 0.6681 & 0.6670 & 0.6438 & 0.6428 & 0.6246 & 0.4173 & 0.1250 \\
\hline
\end{tabular}


Table 12. Evaluative-quantitative indicators and correlated coefficients $\left[{ }^{*} \ldots\right]$ of elements $\mathrm{R} 1 \div \mathrm{R} 8$ from dome K.5

\begin{tabular}{|c|c|c|c|c|c|c|c|c|}
\hline $\begin{array}{c}\text { Dome } \\
\text { K.5 }\end{array}$ & $\begin{array}{c}\text { Element } \\
\text { R1 }\end{array}$ & $\begin{array}{c}\text { Element } \\
\text { R2 }\end{array}$ & $\begin{array}{c}\text { Element } \\
\text { R3 }\end{array}$ & $\begin{array}{c}\text { Element } \\
\text { R4 }\end{array}$ & $\begin{array}{c}\text { Element } \\
\text { R5 }\end{array}$ & $\begin{array}{c}\text { Element } \\
\text { R6 }\end{array}$ & $\begin{array}{c}\text { Element } \\
\text { R7 }\end{array}$ & $\begin{array}{c}\text { Element } \\
\text { R8 }\end{array}$ \\
\hline$D_{a}$ & 17.6589 & 14.8407 & 16.5226 & 16.6548 & 15.7584 & 13.2865 & 8.6187 & 4.2734 \\
\hline$D_{c}$ & 16.8194 & 12.1017 & 12.9806 & 12.6911 & 11.7694 & 9.9772 & 6.7113 & 3.5235 \\
\hline$D_{t}$ & 0.8395 & 2.7390 & 3.5420 & 3.9637 & 3.9889 & 3.3093 & 1.9074 & 0.7498 \\
\hline$K_{c}$ & 0.9525 & 0.8154 & 0.7856 & 0.7620 & 0.7469 & 0.7509 & 0.7787 & 0.8245 \\
\hline$K_{t}$ & 0.0475 & 0.1846 & 0.2144 & 0.2380 & 0.2531 & 0.2491 & 0.2213 & 0.1755 \\
\hline
\end{tabular}

Table 13. Evaluative-quantitative indicators and correlated coefficients $\left[{ }^{*} . ..\right]$ of elements D $\div$ D8 from dome K.5

\begin{tabular}{|c|c|c|c|c|c|c|c|c|}
\hline $\begin{array}{c}\text { Dome } \\
\text { K.5 }\end{array}$ & $\begin{array}{c}\text { Element } \\
\text { D1 }\end{array}$ & $\begin{array}{c}\text { Element } \\
\text { D2 }\end{array}$ & $\begin{array}{c}\text { Element } \\
\text { D3 }\end{array}$ & $\begin{array}{c}\text { Element } \\
\text { D4 }\end{array}$ & $\begin{array}{c}\text { Element } \\
\text { D5 }\end{array}$ & $\begin{array}{c}\text { Element } \\
\text { D6 }\end{array}$ & $\begin{array}{c}\text { Element } \\
\text { D7 }\end{array}$ & $\begin{array}{c}\text { Element } \\
\text { D8 }\end{array}$ \\
\hline$D_{a}$ & 9.4330 & 7.1655 & 7.8426 & 8.7974 & 10.0477 & 11.8891 & 14.0644 & 15.4755 \\
\hline$D_{c}$ & 2.1349 & 4.2997 & 4.3997 & 5.1010 & 6.0052 & 7.3187 & 9.0521 & 9.7271 \\
\hline$D_{t}$ & 7.2981 & 2.8658 & 3.4429 & 3.6964 & 4.0424 & 4.5705 & 5.0123 & 5.7485 \\
\hline$K_{c}$ & 0.2263 & 0.6001 & 0.5610 & 0.5798 & 0.5977 & 0.6156 & 0.6436 & 0.6285 \\
\hline$K_{t}$ & 0.7737 & 0.3999 & 0.4390 & 0.4202 & 0.4023 & 0.3844 & 0.3564 & 0.3715 \\
\hline
\end{tabular}

- Tensile density $D_{t}=\sum\left(\eta_{i}\right)$.

Based on these indicators, new correlated coefficients [*of participation] were derived: Coefficient of compressive activation $K_{c}=D_{c} / D_{a}$ and coefficient of tensile activation $K_{t}=D_{t} / D_{a}$.

\section{CONCLUSION}

The surfaces of influence give a clear idea of the contribution of the load to the final value of effort in the studied element. It is rather easy for the zones of loading to be determined in order to obtain limit value of a normal effort in the selected elements of the structural grid. The constructed IS has shown that the closest to the investigated element area accounts for the largest contribution to the normal effort in the studied element. When the points of application of the forces are far away from the element, attenuation of the influence may be observed. This effect is spatial and depends on the stiffness of the grid structure. Evidently, as IS is more concentrated in the nearby region around an element (with fast "attenuation"), the dome system is more likely to manifest a greater rigidity. New coefficients of compressive 
and tensile activation clearly indicate what part of the loading area of the dome leads to compression or tension in the element. It is easy to follow that for the element R.5 of dome K.1, $77 \%$ of the loading area leads to compressive activation and only $23 \%$ to tensile activation. In other words, when there is uniformly distributed symmetric load, the zones of tensile activation will reduce the normal force in the element by $23 \%$. Similar conclusions can be deduced for each element based on the new coefficients.

\section{REFERENCES}

[1] K. Abedi and G. Parke, Progressive Collapse of Single-Layer Braced Domes, Int. Journal of Space Structures (1996) 11 (3) 291-305.

[2] K. Ikarashi and S. Kato, Elasto-Plastic Dynamic Buckling Analysis of Reticular Domes Subjected to Earthquake Motion, Int. Journal of Space Structures (1997) 12 (3 and 4) 205-215.

[3] D. Dubina, Numerical and Experimental Researches Concerning Two New Systems for Double-Layer Grids, in: Proceedings of the IASS, III, Copenhagen, 1991, pp. 213-220.

[4] V. Gioncu and N. Balut, Instability Behaviour of Single Layer Reticulated Shell, Int. Journal of Space Structures (1992) 7 (4) 243-250.

[5] V. Gioncu, Buckling of Reticulated Shell: State of-the Art, Int. Journal of Space Structures (1995) 10 (1) 1-37.

[6] T. Suzuki, T. Ogawa, and K. Ikarashi, Elasto-Plastic Buckling Analysis of Rigidly Jointed Single Layer Reticulated Domes, Int. Journal of Space Structures (1992) 7 (4) 363-367.

[7] D. Dubina, Computation Models and Numerical Solution Procedures for Nonlinear Analysis of Single Layer Lattice Shell, Int. Journal of Space Structures (1992) 7 (4) 321-333.

[8] R. Levy, A. Hanaor, and N. Rizzuto, Experimental Investigation of Prestressing in Double-Layer Grids, Int. Journal of Space Structures (1994) 9 (1) $21-25$.

[9] H. Agerskov, Optimum Geometry Design of the Space Trusses, Journal of Structural Engineering (1996) 1454-1463.

[10] T. Ueki, S. Kato, I. Kubodera, and Y. Mukaiyama, Study on the Elastic and Elasto-Plastic Buckling Behaviour of Single Layered Domes Composed of Members Having Axial and Bending Springs at Both Ends, in: Proceedings of the IASS, III, Copenhagen, 1991, pp. 93-100. 
[11] I. Stoynova And T. Hristov, Comparative Analysis between Classic Solutions Methods of Flat Slab and Numerical Solutions. Verification of Finite Elements, in: Proceedings of $18^{\text {th }}$ International Symposium MASE'2019, 2-5 October 2019, Ohrid, North Macedonia, on USB, ISBN 978-608-4510-35-2.

[12] I. Stoynova, Comparision of the Shear Bearing Capacity at Centric Punching of the Flat Slab According to the Recommendations of the Codes, in: Proceedings of $14^{\text {th }}$ International Scientific Conference "Planning, Design, Construction and Renewal in the Civil Engineering", 21-23 November 2018, Novi Sad, Serbia, on CD, ISBN 978-86-6022-104-1.

[13] G. Georgiev and St. Tsvetkov, Guide for Design of special Reinforced Concrete Structures According to Eurocodes, Part 1, Chapter 1, General Information for Analysis and Construction of Special Reinforced Concrete Structures, USEA (VSU), Sofia, Bulgaria (2014).

Received August 13, 2020 